\title{
FUT vs. FUE Graft Survival: A Side-by-Side Study of 3 Patients Undergoing a Routine 2,000+ Graft Hair Transplantation*
}

\author{
David Josephitis, DO, FISHRS I Minneapolis, Minnesota, USA I Drjosephitis@shapiromedical.com; \\ Ron Shapiro, MD, FISHRS I Minneapolis, Minnesota, USA
}

*2015 ISHRS Research Grant Recipient Study

\section{INTRODUCTION}

While every patient is unique, the goal of hair transplantation is the same: to provide natural and dense coverage in the recipient area, while taking good care of the donor region. Graft yield (survival) is one of the main determinants of density and coverage. Since the mid-1990s, follicular unit transplantation (FUT) has been the "gold standard" for obtaining grafts. With the use of microscopic follicular unit dissection of the excised strip, viable grafts with low transection rates and high yield are consistently obtained.

An alternative to the FUT method, follicular unit excision (FUE), which uses micro-punches rather than a linear strip, has been developing since the early 2000s. Patient concerns about the linear strip scars and societal trends toward shorter-cropped hairstyles led to the advancement of this method of donor harvesting.

Unfortunately in the early years, FUE results were not as good as FUT results, especially with respect to density and coverage. This was probably due to multiple factors. First, the new technical skill needed to perform the "blind" punch of FUE was difficult to learn and took a long time to master. For many years, there simply was a lack of experienced and skilled physicians practicing the technique. In addition, the grafts produced with FUE tended to have higher transection rates, were skinny and denuded of protective tissue, and were subject to increased forceps trauma during extraction of tethered grafts. Many felt FUE grafts were more "fragile" than FUT grafts due to these various traumas and, therefore, possessed a greater risk of poor survival.'

However, over the years, the FUE technique has been modified and improved. Transection rates have decreased, grafts have more tissue, and forceps extraction is gentler. The survival rate of FUE grafts has improved and many feel the rate is now similar to that of FUT grafts. ${ }^{2,3}$ However, controversy still exists. Is it known for a fact that FUE and FUT graft survival (and the results produced therefrom) are actually identical? This is an important question because many physicians have stopped offering the FUT method altogether, and most new physicians entering the field are learning and providing FUE only.

Only a few studies exist that compare the overall survival of FUE vs FUT grafts. Most of the studies have been small, box studies, working in an isolated area with only a few hundred grafts. ${ }^{4}$ Grafts in small studies like these are usually placed and handled very careful and don't accurately reflect the environment of a real case. This could minimize the difference found between the two techniques that may occur during a larger, full surgery. The use of a full-size case under normal circumstances may better elucidate any shortcom- ings in one technique over the other. The number of grafts done, and time out of the body, handling, and placing grafts, might be more reflective of the conditions that exist during a true full surgery.

This study was designed to compare the overall hair yield and subsequent cosmetic result of the two techniques sideby-side, in the same patient, during a larger-scale, full-size case.

\section{STUDY DESIGN}

Patient selection

Three male patients between the ages of 35 and 60 years were enrolled in the study. All of the patients were Norwood Class $\mathrm{V}$ or greater and none had prior hair transplantation. The front half of their scalps were completely devoid of hair to allow a clean "canvas" for the study (Figure 1). No patients were on hair loss preventative medications.

$$
\text { FIGURE 1. All patients were Norwood Class V or greater. }
$$
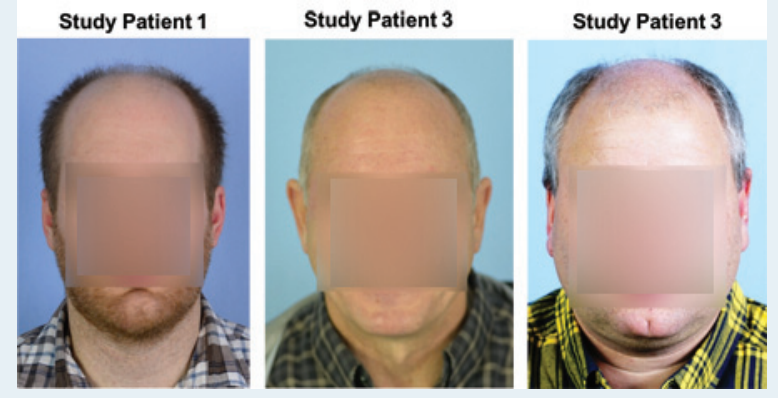

\section{Recipient site design}

A recipient study area was chosen that included the hairline and front third of the balding area (Figure 2). This area was divided in half at the midline sagitally and an equal amount of incisions were made on both sides. The number of incisions made in each half was approximately 1,000 for a total of about 2,000 in the total study area. The right half was delegated for FUT grafts and

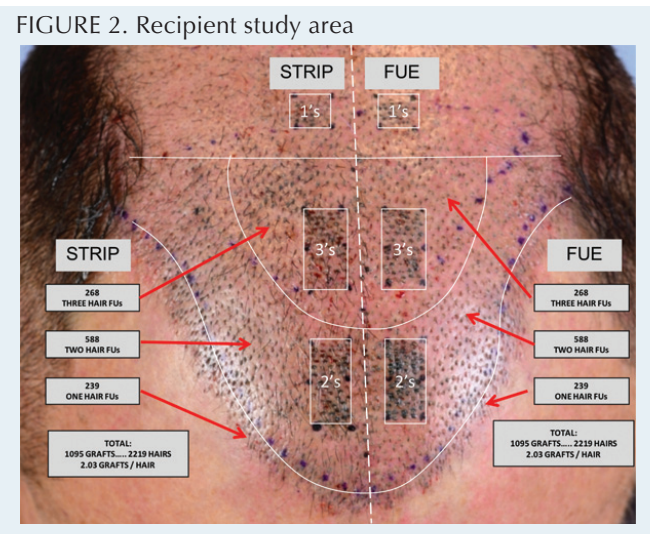
the left half for FUE grafts. All study incisions were made prior to donor harvesting with precut blades at a density of 30 per $\mathrm{cm}^{2}$. For consistency, the incisions were initially 
made with the assistance of a premade ink pattern using a template stamp created by Paco Jimenez. A $0.7 \mathrm{~mm}$ blade was used for 1 -hair grafts and a $0.9 \mathrm{~mm}$ blade was used for both the 2- and 3-hair grafts. The zone just behind the study area was reserved for the extra grafts obtained over the 2,000 needed for the study.

Three pairs of tattooed study boxes, one on either side of the midline, were created within the study area. These boxes were surrounded by recipient incisions, rather than an isolated location. The idea was to place the boxes in an environment more typical of a full surgery. The boxes created included the following:

1. A pair of $1 \times 2 \mathrm{~cm}\left(2 \mathrm{~cm}^{2}\right)$ boxes placed on both sides of the midline slightly behind the hairline for the placement of 2-hair grafts. These boxes would contain 60 grafts each.

2. A second pair of $1 \times 2 \mathrm{~cm}\left(2 \mathrm{~cm}^{2}\right)$ boxes on both sides of the midline, slightly posterior to the first pair, for the placement of 3-hair grafts. These boxes would also contain 60 grafts each.

3. A final pair of $1 \times 1\left(1 \mathrm{~cm}^{2}\right)$ boxes slightly posterior to the second pair for the placement of 1-hair grafts. These boxes would contain only 30 grafts each.

\section{Donor harvesting design}

The donor area was divided in half at the posterior midline (Figure 3). The right half of the donor area was harvested with the FUT (strip) technique in the center of the safe area (level of occipital notch). The same physician with 20+ years of FUT experience removed the strip in all 3 cases. The same technicians with 15+ years of FUT experience microscopically dissected the strip into 1-, 2-, and 3-hair grafts.

FIGURE 3. Donor area divided: right half, FUT; left half, FUE

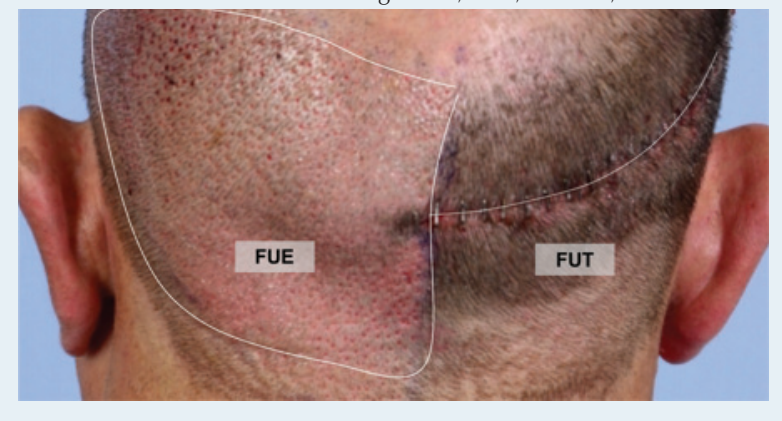

Immediately after the strip incision was closed, the patient's left half of the donor area was harvested with the FUE technique. The extended safe area described by Cole was used. The WAW Hybrid Punch system was used. The same physician with $8+$ years of FUE experience harvested and extracted the grafts in all 3 patients. The grafts were sorted under a microscope into 1-, 2-, and 3-hair grafts.

An important component of this study was ensuring that the exact same number of 1-, 2-, and 3-hair grafts were used on both halves of the study area. There were enough grafts produced on both sides of the donor from each technique to make this fairly easy to do. However, some ex vivo splitting of extra 3-hair FUE grafts was needed in order to make the requisite number and assortment of grafts. The extra grafts harvested above the 2,000 needed for the study were placed posterior to the study area in a natural distribution.

All grafts were stored in cooled Lactated Ringer's solution. No HypoThermosol ${ }^{\circledR}$, PRP, or liposomal ATP was used during the procedure. The reasoning for this was to put maximum equal stress on all grafts to better identify subtle differences in yield that may not be as apparent without stressors.

An attempt was made to ensure time out of the body for both sets of grafts was similar in the following way. FUT strip removal was done first followed immediately by FUE harvesting. This ensured FUE and FUT grafts were being created at approximately the same time. Grafts were also organized by the time they were created. Placing for both sets of graft started simultaneously and the "first graft out, first graft in" approach was used. The total time out of the body was less than 5 hours for all grafts.

\section{Placing design}

All grafts were placed with forceps by the same two technicians, each with 15+ years of experience. One technician placed the FUE side of the recipient, while the other technician placed the FUT side of the recipient. No implanters were used for placing. The reason for this was once again to put maximum equal stress on all grafts to better identify subtle differences in yield that may not be as apparent without stressors.

For simplicity, FUE and FUE grafts were placed on the ipsilateral (same) side from which they were extracted. The sites were stained with Gentian Violet for ease of placement. There was no special treatment given to the placement of grafts in the tattooed boxes.

\section{RESULTS}

\section{Follow-up parameters}

The patients followed up at 4, 8, and 12 months. Measurements taken at these visits included:

- Digital photographs of graft counts and hair yields in study boxes

- Hair Mass Index (HMI) using HairCheck ${ }^{\circledR}$ to measure hair volume. Two locations were used within the study area on each side.

- Gross photography of the patient

- Subjective evaluation from patient

\section{Graft and hair yield}

Table 1 compares FUE vs FUT graft yield per patient within the study boxes on both sides. The total number of grafts placed in the study boxes on each side was 150 . Looking at the first table, there was very little difference noted between the two groups. Grafts on both sides grew consistently well. FUT was slightly better than FUE in 1 patient while FUE was slightly better than FUT in the other 2 patients. However, the difference was very low and never more than $2.7 \%$ in favor of FUE.

\begin{tabular}{|c|c|c|c|}
\hline Patient & FUT & FUE & Difference \\
\hline Patient \#1 & $148 / 150(98.7 \%)$ & $146 / 150(97.3 \%)$ & 1.4\% FUT (over FUE) \\
\hline Patient \#2 & $140 / 150(93.3 \%)$ & $143 / 150$ (95.3\%) & $2.0 \%$ FUE \\
\hline Patient \#3 & $137 / 150(91.3 \%)$ & $141 / 150(94.0 \%)$ & $2.7 \%$ FUE \\
\hline
\end{tabular}


Hair yield is a more informative parameter than graft yield. Grafts can contain any number of hairs (1, 2, or 3 ) and the yield data can be misleading if not carefully examined. This data does not consider the yield of hair within those grafts. Table 2 compares the hair yield per patient within the study boxes on both sides. The total number of hairs from the single, double, and triple graft study boxes were counted showing a total of 330 total hairs placed in the study boxes on each side. The findings were similar to those of graft yield with FUT being slightly better in 1 patient and FUE being slightly better in the other 2 patients. The difference was still low, but a little more obvious with hair yield than with graft yield, reaching $9.4 \%$ in 1 of the 3 patients.

Table 2. Hair Yield per Patient

\begin{tabular}{|l|c|c|c|}
\hline Patient & FUT & FUE & Difference \\
\hline Patient \#1 & $242 / 330(73.3 \%)$ & $240 / 330(72.7 \%)$ & $\mathbf{0 . 6 \%}$ FUT (over FUE) \\
\hline Patient \#2 & $244 / 330(73.9 \%)$ & $272 / 330(82.4 \%)$ & $\mathbf{8 . 5 \%}$ FUE \\
\hline Patient \#3 & $244 / 330(73.9 \%)$ & $275 / 330(83.3 \%)$ & $9.4 \%$ FUE \\
\hline
\end{tabular}

Table 3 shows the difference in graft and hair yield when the data from all three patients is combined. This is probably the best parameter to look at. Again, there was very little difference in the survival of grafts in both groups. For graft yield, the difference between the two groups was about $1 \%$ in favor of FUE. For hair yield, the difference was just slightly higher at about $6 \%$ in favor of FUE.

Table 3. Total Graft and Hair Yields

\begin{tabular}{|l|c|c|c|}
\hline \multicolumn{1}{|c|}{ Combined Results } & FUT & FUE & Difference \\
\hline Combined Graft Yield & $\mathbf{4 2 5} / 450(\mathbf{9 4 . 4 \% )}$ & $\mathbf{4 3 0 / 4 5 0}(\mathbf{9 5 . 6 \% )}$ & $\mathbf{1 . 2 \%}$ FUE (over FUT) \\
\hline Combined Hair Yield & $\mathbf{7 3 0 / 9 9 0}(\mathbf{7 3 . 7 \% )}$ & $\mathbf{7 8 7 / 9 9 0}(\mathbf{7 9 . 5 \% )}$ & $\mathbf{5 . 8 \%}$ FUE \\
\hline
\end{tabular}

From a practical standpoint, due to the small size of this study, no hard conclusion can be drawn that one technique has a better survival rate than the other on a consistent basis. However, since the question that we were most concerned about was whether FUE graft survival was less than FUT grafts, the results are interesting since in this case FUE graft survival seemed to be slightly better.

\section{Hair Mass Index}

Table 4 compares the $\mathrm{HMI}$ on each side of the study area. Unfortunately, only 2 patients were available for this measurement. The measurements were made at two locations

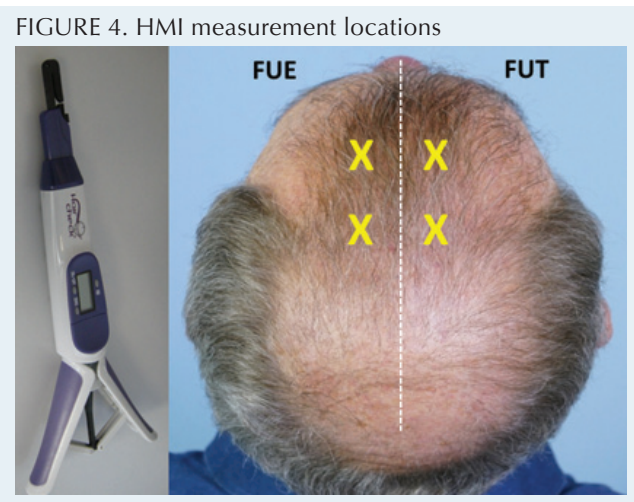
on both sides of the study area (the anterior and posterior parts of the frontal zone (Figure 4). The HMI was almost identical in the FUT side compared to the FUE side of the study.

Table 4. Hair Mass Index with HairCheck

\begin{tabular}{|l|l|l|}
\hline Patient & Frontal (21) / Midscalp (22) & Frontal (21) / Midscalp (23) \\
\hline Patient \#2 & Frontal (27)/ Midscalp (27) & Frontal (27) / Midscalp (26) \\
\hline Patient \#3 & Frontal (24) / Midscalp (25) & Frontal (24) / Midscalp (25) \\
\hline Average for Both Patients & &
\end{tabular}

\section{Gross photography}

Gross photography was done on patients at various intervals throughout the study (Figures 5). There was no difference in the speed of growth of one group versus the other. Also, the final 12-month follow-up photos show no large difference between the groups.

FIGURE 5. One-year post-op, gross photography shows no difference in speed of growth between the techniques.

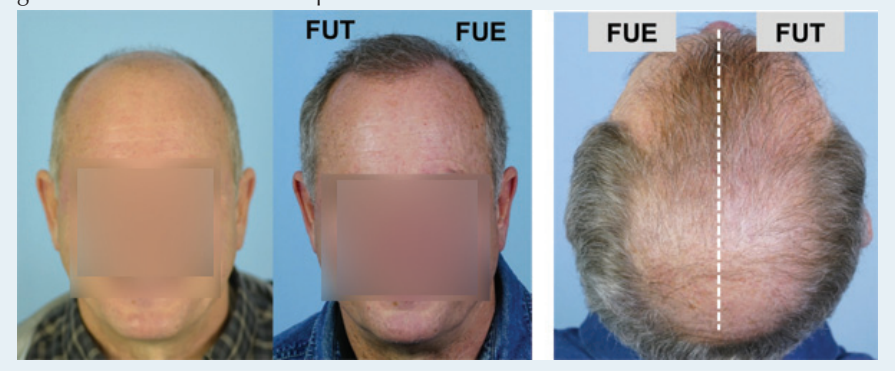

\section{Subjective evaluation from patients}

All patients felt that the results with respect to rate of growth, feel, and appearance of fullness were the same on both sides.

\section{DISCUSSION \& CONCLUSION}

The ability to meet patient expectations of density and coverage is one of the most important goals in hair transplantation. The ability to produce density and coverage is primarily determined by two things:

1. The amount of donor that can be safely harvested from a patient per session and over the life of the patient.

2. The survival of those grafts that are harvested and transplanted.

For many years, there has been controversy over the survival of FUE vs FUT grafts. Many have felt that FUE grafts are "more fragile" than FUT grafts and have a higher risk of poor survival. This was true early in the development of FUE for reasons stated earlier in this paper. But with the improvements that have occurred in the FUE technique over the past 5 years, this question has become more controversial. Studies to address this question have been small and primarily of the isolated box study method. This type of study does not mimic the true environment and stressors grafts are subjected to during the course a full transplant.

The purpose of our study was to see if FUE graft survival, with modern techniques, is indeed less than the survival of FUT grafts. We wanted to do this in the environment of a true full surgery in order to better imitate the actual environment and stressors to which grafts are exposed during a full surgery. We felt this would better identify subtle differences in survival between the two techniques than the less realistic, gentle environment of an isolated box study. We did the hair counts in study boxes that were placed in the center of a full hair transplant, surrounded by other grafts. The same routine placing and care was done in the study boxes as would be done in the rest of case. The tattooed boxes were only created as a way to standardize and adequately compare both sides.

We also decided not to use bio-enhancers (e.g., ACell, 
liposomal ATP, HypoThermosol, PRP) or implanters for a similar reason. We felt that by not using these adjuncts, a greater stress would be equally placed on both types of grafts, and we'd be better able to identify subtle differences in yield that may not be as apparent without stressors.

Four variables were looked at in this study to compare the yield of FUT vs FUE grafts: graft and hair yield, HMI, gross photography, and patient subjective comments. For all practical purposes, FUE and FUT were equal with all parameters. Although FUE seemed to have a very small edge with respect to graft and hair yield counts, the study was too small to draw any conclusions. However, the fact that FUE grafts performed as well as or a little better in all parameters seems to provide good evidence that, at a minimum, FUE graft survival is not worse than FUT graft survival. In addition, with the addition of implanters and bio-enhancers, the survival of FUE grafts could quite possibly be even better than in this study

It should be mentioned that some of the same factors that plagued early FUE results can still cause issues today. With inexperienced technicians and improper graft handling, the results of this study could have been vastly different. Physicians need more than a modicum of experience in both techniques to deliver results like these.
I would like to mention one final point. Graft survival is only one of two important variables that ultimately determine the ability to produce density and coverage in a patient. The "number of grafts" that can be obtained over the life of the patient is a second, very important factor. We still do not know the difference in the number of grafts we can obtain on the same patient if they were approached with FUE only, FUT only, or FUE + FUT combination. I believe understanding this better is the final cog in the controversy over the ability of different approaches to produce the best results. This is an extremely important question to answer, especially with the increasing trend toward FUE-only practices and new physicians coming into the field learning only FUE.

\section{References}

1. Beehner, M. A comparison of hair growth between follicular unit grafts trimmed "skinny" vs. "chubby." Hair Transplant Forum Int'l. 1999; 9(1):16.

2. Pathomvanich D. FUT fights back. Hair Transplant Forum Int'l. 2015; 25(5):177, 183-184.

3. Various authors. Physicians sound off: FUT vs. FUE. Hair Transplant Forum Int'l. 2015; 25(5):185-187.

4. Beehner, M. FUE vs. FUT-MD: study of 1,780 follicles in four patients. Hair Transplant Forum Int'l. 2016; 26(4):160-161.

\section{FUE Instruments}

You asked. We listened. We developed.

\section{Steel Blunt Punches from Harris F.U.E. Instruments}

THE ADVANTAGE:

- Approved for up to 40,000 grafts with one punch

- New $6 \mathrm{~mm}$ depth for all follicle lengths

- HEX vibration separates follicle from skin

- Low transection rate of $2.8 \%$ *

- Reusable punch

- Flush port

- Proven for body hair

- Ideal punch for beginners and experts

\section{To pre-order, contact us:}

Phone Veronica Melero at 720.536.4897

Email vmelero@harrisfueinstruments.com Visit us online: harrisfueinstruments.com

"In a clinical study by Dr. Harris in over 150 patients and more than 100,000 harvested grafts. General user transection rates may differ 